\title{
The July Effect in Radical Cystectomy: Mortality, Morbidity, and Efficiency
}

\author{
Keegan Zuk, Derek Jensen, Jessie Gills, Hadley Wyre, Jeffrey M. Holzbeierlein, \\ Ernesto Lopez-Corona and Eugene K. Lee* \\ Department of Urology, University of Kansas Medical Center, Kansas City, KS, USA
}

\begin{abstract}
.
Background: The "July effect" is the potential effect that new and recently promoted residents have on patient care during the first months of the academic year. Literature suggests that the July effect may worsen patient outcomes and lead to systemic inefficiencies.

Objective: We evaluate the July effect on mortality, morbidity, and efficiency outcomes in patients undergoing radical cystectomy.

Methods: A chart review was performed in patients who underwent radical cystectomy between January 2008 and April 2012. Demographic information was abstracted from patient charts and outcomes compared between operations performed in July, September, and November (first month of each resident's university rotation) to the remainder of the year. Outcomes of interest included mortality, complications, and markers of efficiency (operative time, length of hospital stay, and estimated blood loss).

Results: Two hundred and fifty one patients were included in the analysis. There were no major differences in mortality or morbidity between the July, September, November group and the rest of the year. Multivariable analysis demonstrates a trend for operations performed in the months of July, September, and November to be associated with longer OR times $2.06(0.99-4.27), p=0.053$. Length of hospital stay and estimated blood loss were no different between the two groups.

Conclusions: These data demonstrate no increase in mortality or morbidity during the early academic period. Additionally, while there is a trend for OR time to be longer in the early group, length of hospital stay and estimated blood loss were no different. These data may be used as an impetus to continue to investigate technical/clinical teaching practices, strategies to assess resident progression, and to initiate protocols to support residents early in the academic year in efforts to prevent inefficiencies.
\end{abstract}

Keywords: Radical cystectomy, July effect, efficiency markers

\section{INTRODUCTION}

Every year, the month of July marks a transition in academic medical centers around the country. Senior residents graduate and are replaced by their junior counterparts who are, in turn, replaced by

\footnotetext{
${ }^{*}$ Correspondence to: Eugene K. Lee, Department of Urology, University of Kansas Medical Center, 3901 Rainbow Boulevard, MS 3016, Kansas City, KS 66160, USA. Tel.: +1 913588 7564; Fax: +1 913588 6668; E-mail: elee@kumc.edu.
}

new medical school graduates. The potential effect of this transition on patient care is often referred to as the "July effect." This effect has long been a subject of apprehension both in patient and physician communities and has been investigated as a potential contributor to an increase in systemic inefficiencies in the medical system [1-5], medical errors [6-8], perioperative complications $[1,2,9]$ and even perioperative mortality $[1,9]$. In one of the more striking examples, Englesbe et al. analyzed a 20,254 patient 
sample from the ACS-NISQIP (American College of Surgeon - National Surgical Quality Improvement Project) database and found that patients who underwent surgeries in the first half of the academic year had a $41 \%$ relative increase in mortality compared to the second $(2.2 \%$ v $1.7 \%, p=0.009)$. Such studies, especially those drawing from a largely academic patient cohort, call into question the role of inexperienced residents in complex surgical cases early in the academic year [1].

Radical cystectomy (RC) is one such complex and technically challenging urologic operation. It is performed on patients with significant comorbidities whose post-operative course requires attentive and skilled management. Contemporary, retrospective data demonstrate that these patients have high rates of complications $(45.7-68 \%)[10,11]$, high peri-operative mortality (1.7-6.9\%) [10-12], and significant 90-day readmission rates (27\%) [12]. These factors make RC an ideal surgical procedure on which to highlight the potential weaknesses of less experienced trainees in the surgical and medical management of patients. While there are data to refute the existence of a July effect on the morbidity and mortality of radical cystectomy patients [13], to date, there is no study that investigates the July effect on markers of healthcare efficiency and technical skills in RC.

In this study, we sought to examine the possible effect that resident teams composed of newly promoted residents would have on the outcomes and overall care of patients who underwent a RC for bladder cancer. We hypothesized that while there might not be detectable differences in peri-operative mortality or complications, there may be a difference in markers of efficiency and technical skill using markers such as operating room time (OR time), length of hospital stay (LOS), and estimated blood loss (EBL).

\section{METHODS}

After Institutional Review Board approval (Study 1328) was obtained from the University of Kansas Medical Center, we performed a retrospective chart review of patients who underwent RC for a diagnosis of histologically confirmed bladder cancer from January 2008 to April 2012. Patients who underwent RC for non-bladder cancer indications were excluded. Patients who underwent both roboticassisted laparoscopic and open cystectomies were included in the analysis. The decision of ileal conduit or orthotopic neobladder diversion was made at the discretion of the attending surgeon in conjunction with the patient. The operations were performed by one of three fellowship trained urological oncologists at a single academic medical center. Staging was performed by board certified pathologists in accordance with the American Joint Committee on Cancer's AJCC Cancer Staging Manual [14].

Preoperative demographic information including gender, race, age and body mass index (BMI) was abstracted from patient's records, along with pathologic diagnosis/stage, date of operation, receipt of neoadjuvant chemotherapy, operative approach, and diversion type. Outcome variables analyzed included overall survival, 90-day survival, 30 and 90-day post-operative complications (Clavien-Dindo Classification), operating room time (OR time), length of hospital stay (LOS), and estimated blood loss (EBL) [15].

Our institutional training program accepts three residents per year, each performing rotations in twomonth blocks. In our analysis, we compared data from the first month of each resident's first rotation on the University urology service during the academic year to the data from the rest of the year. Therefore, we compared data from patients who underwent a radical cystectomy for bladder cancer in the months of July, September, and November to patients undergoing RC during the other 9 months of the year.

The data were collected and managed using REDCap (Research Electronic Data Capture) hosted at the University of Kansas Medical Center to ensure protection of patient information [16]. Data analysis was completed using SPSS v22.0 (SPSS, Chicago, IL, USA). Descriptive statistics were utilized to summarize the demographic characteristics. Variable data was presented as percentage of total for categorical data and median values with $25-75$ th percentiles for continuous variables. All categorical variables were compared using Chi-square analysis and Mann-Whitney U test for continuous variables. For univariate analysis, continuous variables were dichotomized by the median value. For multivariate analysis we used logistic regression analysis using the standard method and OR time, LOS and EBL were dichotomized according to median values of the entire cohort. Odds ratios and $95 \%$ confidence intervals (CI) were derived from the analysis. Survival data were summarized using Kaplan-Meier plots and compared using log-rank test. A $p$ value $<0.05$ was considered statistically significant. 


\section{RESULTS}

Between January 2008 and April 2012, 264 patients underwent cystectomy. Thirteen patients underwent surgery for non-bladder cancer indications (gynecologic cancer, gastrointestinal cancer, cystectomy for benign disease). The study cohort consisted of 251 patients, of whom the median age was 69 and $189(75 \%)$ were men. Table 1 describes patient demographics and clinical variables considered in the analysis. A majority of patients underwent an open surgical procedure $(201,80 \%)$ while the remainder underwent a robotic procedure. Ileal conduit diversion was performed on 152 patients $(61 \%)$ and neobladder diversion was performed on 95 patients (38\%). Neoadjuvant chemotherapy was received by 78 patients $(31 \%)$. Sixty patients $(24 \%)$ underwent a radical cystectomy in the months of July, September and November, and 191 patients (76\%) underwent RC during the other 9 months. One hundred thirty-two patients experienced a complication (54\%) within 90 days, with 57 (23\%) experiencing a major complication (Clavien-Dindo grade III-V) within 90 days. For the entire cohort, the median OR time was 331 minutes (25th/75th: 285/409 minutes), the median LOS was 7 days (25th/75th: 6/9 days), with a median EBL of $600 \mathrm{~mL}$ (25th/75th: 350/850 mL).
The median follow-up for the cohort was 25.3 months at which time the overall survival was not statistically different between the early surgery group versus the rest of the year $(p=0.32, \log$-rank test) (Fig. 1). Additionally, there were a total of nine deaths within 90 days of RC. One death occurred $(1 / 60-2 \%)$ in the July, September, November group and eight deaths $(8 / 191-4 \%)$ occurred in the rest of the year group $(p=0.37)$.

On univariate analysis (Table 2), there was no difference in rates of overall or major 90-day complications between the surgeries performed in the months of July, September, and November and the surgeries performed during the rest of the year. Additionally, univariate analysis found no significant difference in OR time, LOS, or EBL when comparing the two groups of interest. As expected, variables that were associated with increased OR time included gender (female 360 minutes vs. male 324 minutes, $p=0.027$ ), operative approach (open 316 minutes vs. robotic 414 minutes, $p<0.001)$, age $(\leq 69348 \mathrm{~min}$ utes vs. $>69315$ minutes, $p=0.003$ ) and diversion type (Ileal conduit 316 minutes vs. neobladder 353 minutes, $p=0.036$. Additionally, patients with higher BMI ( $\leq 29$ vs. $>29$ ) were found to have increased length of stay ( 8 vs. 7, $p=0.037$ ). Variables affecting EBL included gender (female $750 \mathrm{~mL}$ vs. male

Table 1

Patient characteristics

\begin{tabular}{|c|c|c|c|c|}
\hline Total number of pts. & $\begin{array}{c}\text { Total Patients } \\
251\end{array}$ & $\begin{array}{c}\text { July/Sep/Nov } \\
60\end{array}$ & $\begin{array}{c}\text { Rest of Year } \\
191\end{array}$ & $p$-value \\
\hline \multicolumn{5}{|l|}{ Age (years) } \\
\hline Median & 70 & 68 & 70 & \multirow[t]{2}{*}{0.71} \\
\hline $25-75$ percentile & $62-76$ & $62-77$ & $62-76$ & \\
\hline \multicolumn{5}{|l|}{ BMI } \\
\hline Median & 29 & 29 & 28 & \multirow[t]{2}{*}{0.76} \\
\hline $25-75$ percentile & $26-32$ & $26-32$ & $26-32$ & \\
\hline \multicolumn{5}{|l|}{ Gender } \\
\hline Male (\%) & $189(75)$ & $47(78)$ & $142(74)$ & \multirow[t]{2}{*}{0.53} \\
\hline Female (\%) & $62(25)$ & $13(22)$ & $49(26)$ & \\
\hline \multicolumn{5}{|l|}{ Surgery type } \\
\hline Open $(\%)$ & $201(80)$ & $48(80)$ & $153(80)$ & \multirow[t]{2}{*}{0.99} \\
\hline Robotic (\%) & $50(20)$ & $12(20)$ & $38(20)$ & \\
\hline \multicolumn{5}{|l|}{ Diversion } \\
\hline Ileal Conduit (\%) & $152(61)$ & $31(53)$ & $121(64)$ & \multirow[t]{3}{*}{0.17} \\
\hline Neobladder $(\%)$ & $95(38)$ & $27(47)$ & $68(36)$ & \\
\hline Missing (\%) & $4(2)$ & & & \\
\hline \multicolumn{5}{|c|}{ Neoadjuvant Chemotherapy } \\
\hline No $(\%)$ & $173(69)$ & $42(70)$ & $131(69)$ & \multirow[t]{2}{*}{0.84} \\
\hline Yes $(\%)$ & $78(31)$ & $18(30)$ & $60(31)$ & \\
\hline \multicolumn{5}{|l|}{ pStage } \\
\hline $\mathrm{T} 0$, Ta, Cis & $40(16)$ & $12(20)$ & $28(15)$ & \multirow[t]{5}{*}{0.6} \\
\hline $\mathrm{T} 1$ & $50(20)$ & $8(13)$ & $42(22)$ & \\
\hline $\mathrm{T} 2$ & $60(24)$ & $15(25)$ & $45(24)$ & \\
\hline $\mathrm{T} 3$ & $70(28)$ & $18(30)$ & $52(27)$ & \\
\hline $\mathrm{T} 4$ & $31(12)$ & $7(12)$ & 24 (13) & \\
\hline
\end{tabular}




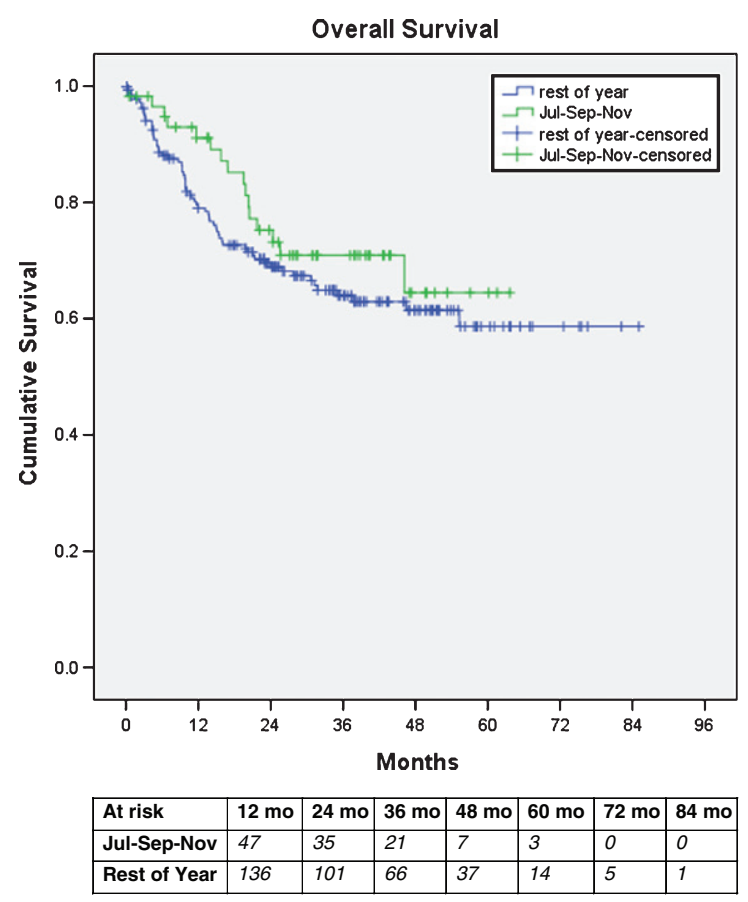

Fig. 1. Kaplan Meier Curve of Overall Survival.

$580 \mathrm{~mL}, p<0.001$ ) and operative approach (open $600 \mathrm{~mL}$ vs. robotic $400 \mathrm{~mL}, p=0.003$ ).

For multivariable analysis, we used logistic regression analysis using the standard method. All variables used in the univariate analysis were carried over for multivariable analysis. OR time, LOS and EBL were dichotomized according to median values of the entire cohort. Female gender, BMI, and robotic surgery all demonstrated an association with prolonged OR times (Table 3). There was a trend for patients undergoing surgery in July/Sep/Nov to have an association with longer OR times (OR of 2.06, $p=0.053$ ) and a trend for older patients to have an association with shorter OR times (OR 0.96, $p=0.052$ ). On multivariable analysis for length of stay, only increasing BMI demonstrated an association with increased hospital stay (OR 1.05, $p=0.047$, Table 4). Additionally, female gender (OR 1.94, $p=0.035$ ) and patients undergoing open surgery (OR 1.94, $p=0.035)$ had an association with increased EBL (Table 5).

\section{DISCUSSION}

Our results are in agreement with previous studies that suggest that patients undergoing radical cystectomy are not subjected to the July effect in terms of clinically significant factors such as increased morbidity or mortality. When factors to elucidate assessment of clinical care, efficiency and technical skill with the addition of a new team member were examined, the complexity and dynamic nature of bladder cancer surgery and care supervened. While the effect may manifest itself in measures of healthcare efficiency such as OR time and the length of hospital stay, better markers of healthcare efficiency and technical skill need to be surmised. Multivariable analysis showed that radical cystectomies performed in the early training window had a trend for association with increased OR time. However, the data also demonstrate that the length of hospital stay and EBL was no different in July, September, and November compared to other months of the year. Again, highlighting mixed results of efficiency during residents first months.

Reassuringly, our data also supports the hypothesis that there is no significant difference in mortality or complication rates between the two groups in our cohort. The literature has previously described that radical cystectomies performed in the month of July do not result in a significant increase in perioperative complications or peri-operative mortality. Using data from the Nationwide Inpatient Sample (NIS) database over 10 years, Ravi et al. showed that there was no increase in mortality associated with 8 major oncologic surgeries, including cystectomies, during the month of July [13]. Several other studies have similarly failed to show the presence of a July effect on mortality of patients following orthotopic liver transplants [17] and laparoscopic gastric bypass [3].

One could reason that decades of practice integrating new residents into operative performance and patient care have helped shape systems to create failsafes and checks to prevent a major negative impact on patients. It would follow that one is more likely to detect a July effect in more subtle outcome measures, especially those that are possible indicators of efficiency. Indeed, previous studies have documented July increases in LOS for patients undergoing spinal surgery [2] and appendectomies [4], and increases in operative time for inpatient surgeries [1], specifically gastric bypass [3] and cardiac surgeries [5]. In this study, we found that one proxy marker of healthcare efficiency, OR time, was increased while novice trainees are operating on and caring for these patients. Herein, we provide the first description of the July effect on measures of efficiency in patients undergoing radical cystectomies. 
Table 2

Univariate analysis according to groups and outcomes of interest

\begin{tabular}{|c|c|c|c|c|c|}
\hline $\begin{array}{l}\text { Variables } \\
\text { (Total pts) }\end{array}$ & $\begin{array}{c}\text { Any } \\
\text { complication } \\
(\%)\end{array}$ & $\begin{array}{c}\text { Major } \\
\text { complication } \\
(\%)\end{array}$ & $\begin{array}{c}\text { OR time } \\
\text { (Median and } \\
\text { 25-75th percentile } \\
\text { Minutes) }\end{array}$ & $\begin{array}{c}\text { EBL } \\
\text { (Median and } \\
\text { 25-75th } \\
\text { percentile mL) }\end{array}$ & $\begin{array}{c}\text { LOS } \\
\text { (Median and } \\
\text { 25-75th } \\
\text { percentile Days) }\end{array}$ \\
\hline \multicolumn{6}{|l|}{ Gender } \\
\hline Female (69) & $29(47)$ & $11(18)$ & $360(303-434)$ & $750(500-1100)$ & $7(6-9)$ \\
\hline \multirow[t]{2}{*}{ Male (189) } & $103(55)$ & $46(24)$ & $324(282-393)$ & $580(350-800)$ & $7(6-9)$ \\
\hline & $p=0.29$ & $p=0.28$ & $p=0.027$ & $p=0.003$ & $p=0.45$ \\
\hline \multicolumn{6}{|l|}{ Age } \\
\hline$\leq 69(120)$ & $67(44)$ & $22(18)$ & $348(302-415)$ & $600(375-900)$ & $7(6-9)$ \\
\hline \multirow[t]{2}{*}{$>69(131)$} & $65(50)$ & $35(27)$ & $315(272-382)$ & $550(300-800)$ & $7(6-9)$ \\
\hline & $p=0.32$ & $p=0.11$ & $p=0.003$ & $p=0.081$ & $p=0.36$ \\
\hline \multicolumn{6}{|l|}{ BMI } \\
\hline$\leq 29(145)$ & $65(45)$ & $30(21)$ & $327(282-414)$ & $600(330-855)$ & $7(6-9)$ \\
\hline \multirow[t]{2}{*}{$>29(106)$} & $67(63)$ & $27(25)$ & $332(289-399)$ & $600(350-800)$ & $8(6-11)$ \\
\hline & $p=0.004$ & $p=0.37$ & $p=0.92$ & $P=0.78$ & $P=0.037$ \\
\hline \multicolumn{6}{|l|}{ Surgery type } \\
\hline Open (201) & $106(52)$ & $41(20)$ & $316(272-367)$ & $600(400-900)$ & $7(6-9)$ \\
\hline \multirow[t]{2}{*}{ Robotic (50) } & $26(52)$ & $16(32)$ & $414(369-470)$ & $400(250-600)$ & $7(6-9)$ \\
\hline & $p=0.92$ & $p=0.08$ & $p<0.001$ & $P<0.001$ & $p=0.18$ \\
\hline \multicolumn{6}{|l|}{ Diversion } \\
\hline Ileal Conduit (152) & $78(51)$ & $36(24)$ & $316(272-372)$ & $600(300-900)$ & $8(6-9)$ \\
\hline \multirow[t]{2}{*}{ Neobladder (95) } & $52(55)$ & $20(21)$ & $353(300-434)$ & $600(350-800)$ & $7(6-9)$ \\
\hline & $p=0.87$ & $p=0.88$ & $p=0.036$ & $p=0.94$ & $p=0.39$ \\
\hline \multicolumn{6}{|c|}{ Neoadjuvant Chemotherapy } \\
\hline No (173) & $93(54)$ & $42(24)$ & $324(282-398)$ & $600(350-800)$ & $7(6-9)$ \\
\hline \multirow[t]{2}{*}{ Yes (78) } & $39(50)$ & $15(19)$ & $345(285-414)$ & $600(350-900)$ & $7(6-9)$ \\
\hline & $p=0.58$ & $p=0.37$ & $p=0.28$ & $p=0.52$ & $p=0.93$ \\
\hline \multicolumn{6}{|l|}{ Month of Surgery } \\
\hline July/Sep/Nov (60) & $34(57)$ & $17(28)$ & $334(284-410)$ & $600(350-850)$ & $7(6-9)$ \\
\hline \multirow{2}{*}{ Rest of year (191) } & $98(51)$ & $40(21)$ & $325(287-382)$ & $525(300-950)$ & $7(6-9)$ \\
\hline & $p=0.47$ & $p=0.23$ & $p=0.91$ & $p=0.41$ & $p=0.14$ \\
\hline
\end{tabular}

Table 3

Multivariable analysis by logistic regression for Operating Room time (OR time). OR time was dichotomized by the median ( 331 minutes) and odds ratios are demonstrated for greater versus lesser than median value

\begin{tabular}{lc}
\hline OR Time (minutes) & OR $(95 \% \mathrm{CI})$ \\
& $p$ value \\
\hline Female vs Male & $2.55(1.28-5.08)$ \\
& $p=0.007$ \\
BMI & $1.06(1.04-1.11)$ \\
& $p=0.034$ \\
Type of Surgery Open vs Robotic & $0.059(0.02-0.15)$ \\
& $p=<0.001$ \\
July/Sep/Nov vs rest of year & $2.06(0.99-4.27)$ \\
Age & $p=0.053$ \\
& $0.96(0.96-1)$ \\
& $p=0.052$ \\
\hline
\end{tabular}

Other variables included in the analysis were type of diversion, use of neoadjuvant chemotherapy, and pStage. Variables that were NS were not included in the table.

One potential implication of this research is impact the July effect might have on the cost of healthcare. In an attempt to characterize the cost of increased operative time, Macario et al. estimated that it
Table 4

Multivariable analysis by logistic regression for length of hospital stay (LOS). LOS was dichotomized by the median (7 days) and odds ratios are demonstrated for greater versus lesser than median value

\begin{tabular}{lc}
\hline LOS (days) & OR $(95 \% \mathrm{CI})$ \\
& $p$ value \\
\hline BMI & $1.05(1.001-1.11)$ \\
& $p=0.047$ \\
\hline
\end{tabular}

Variables also included in the analysis were gender, age, type of surgery, type of diversion, use of neoadjuvant chemotherapy, pStage, surgery done on July/Sep/Nov. Variables that were NS were not included in the table.

costs $\$ 62 / \mathrm{min}$ for time in the OR during 2005 [18]. In a similar manner, many have tried to estimate the cost of inpatient admissions. One such study found that in the mean cost of hospital stays across conditions was $\$ 2,157 /$ day in the U.S. in 2015 [19]. When addressing post-operative care and LOS inefficiencies, one promising solution to the dilemma is the development and implementation of enhanced recovery after surgery (ERAS) pathways: multi- 
Table 5

Multivariable analysis by logistic regression for estimated blood loss (EBL). EBL was dichotomized by the median $(600 \mathrm{~mL})$ and odds ratios are demonstrated for greater versus lesser than median value

\begin{tabular}{lc}
\hline EBL $(\mathrm{mL})$ & OR $(95 \% \mathrm{CI})$ \\
& $p$ value \\
\hline Female vs Male & $1.94(1.046-3.61)$ \\
& $p=0.035$ \\
Type of surgery Open vs Robotic) & $2.95(1.38-6.18)$ \\
& $p=0.005$ \\
\hline
\end{tabular}

Variables also included in the analysis were BMI, age, type of diversion, use of neoadjuvant chemotherapy, pStage, surgery done on July/Sep/Nov. Variables that were NS were not included in the table.

modal perioperative clinical pathways that provide structured algorithms to optimize patient care. There is evidence that the implementation of ERAS pathways in colorectal surgery and radical cystectomy patients has led to reductions in cost of patient care [20], length of hospital stay [20-22] and even postoperative complications [20, 21, 23, 24]. One could reason that this type of framework would be particularly effective for residents who lack a wealth of clinical experience on which to base their clinical decisions. Evidence also exists to guide attempts to improve intra-operative efficiency. Systems for graduated mastery of operative skills using a step-by-step approach have been published for urologic surgery [25]. Taking time to gradually involve trainees in a controlled manner could help increase operative efficiency.

Our results are not without limitations. The study was performed retrospectively and with data from a single institution with small numbers. A multicenter trial may be more helpful in determining whether these inefficiencies are unique to one health system or whether they are seen across systems. Our markers of efficiency, while intuitive and not novel, still need to be verified and expounded upon. Nevertheless, the fact that these markers of efficiency have been well documented in other surgical patients at various institutions as described above is supportive of our hypothesis. Second, although our outcomes of interest (LOS, OR time, EBL) are not direct correlates, they serve as proxy markers of the efficiency of healthcare delivery of the surgical team, where the addition of new members goes further than the addition of a new resident with each resident consisting of a unique clinical and technical skill set. We acknowledge that there is variability within trainees of the same level which is unexplored in our analysis. In the setting of a retrospective chart review, we are limited to using such markers because they are the most available way for the investigators to measure efficiency. Clearly, there are other confounding factors that may impact our outcomes of interest such as the difficulty of each case, medical comorbidities of the patient, past surgical history, the experience of the OR team as a whole or individual. Despite these limitations, we did control for the most common contributing factors such as diversion, gender, surgical approach, previous chemotherapy, BMI and age. Measurements of real time clinical and technical skills are difficult to measure retrospectively. However, our proxy markers of efficiency show greater variability during a resident's first month during a new role than during the remainder of the year.

These data are meant to create awareness for academic medical centers to help improve efficiency, optimize resident teaching, and enhance patient safety. In the end, the authors would contend that while the realities of modern healthcare dictate that we do our best to maximize the efficiency of the care we delivery, we must accept inefficiency as an inherent aspect of training process. Identifying areas of inefficiency will hopefully allow us to better objectively intervene without negatively impacting the quality of surgical training or quality of care these patients receive.

\section{CONCLUSION}

These data provide evidence that there may be a "July effect" on operative and healthcare efficiency in patients undergoing radical cystectomies in academic medical centers. These findings likely speak to the complexity of the execution of the radical cystectomy and the post-operative care for patients undergoing the procedure. These data also provide a strong impetus to investigate the July effect on operative and healthcare efficiency in surgical patients using larger, nationwide databases. Finally, the data presented further challenge resident training programs to develop systems to help guide and support residents as they grow in experience, both as clinicians and as surgeons.

\section{FINANCIAL DISCLOSURES}

No. 


\section{REFERENCES}

[1] Englesbe MJ, et al. Seasonal variation in surgical outcomes as measured by the American College of Surgeons-National Surgical Quality Improvement Program (ACS-NSQIP). Ann Surg 2007;246(3):456-62; discussion 463-5.

[2] Nandyala SV, et al. Perioperative characteristics and outcomes of patients undergoing anterior cervical fusion in July: Analysis of the July effect. Spine (Phila Pa 1976) 2014; 39(7):612-7.

[3] Krell RW, et al. Effects of resident involvement on complication rates after laparoscopic gastric bypass. J Am Coll Surg 2014;218(2):253-60.

[4] Yaghoubian A, et al. July effect and appendicitis. J Surg Educ 2010;67(3):157-60.

[5] Bakaeen FG, et al. The July effect: Impact of the beginning of the academic cycle on cardiac surgical outcomes in a cohort of 70,616 patients. Ann Thorac Surg 2009;88(1): 70-5.

[6] Inaba K. Complications and death at the start of the new academic year: Is there a July phenomenon? Journal of trauma: Injury, Infection, and Critical Care 2010;68(1):19-22.

[7] Haller G, et al. Rate of undesirable events at beginning of academic year: Retrospective cohort study. BMJ 2009;339: b3974.

[8] Phillips DP, Barker GE. A July spike in fatal medication errors: A possible effect of new medical residents. J Gen Intern Med 2010;25(8):774-9.

[9] Dasenbrock HH, et al. The impact of July hospital admission on outcome after surgery for spinal metastases at academic medical centers in the United States, 2005 to 2008. Cancer 2012;118(5):1429-38.

[10] Donat SM, et al. Potential impact of postoperative early complications on the timing of adjuvant chemotherapy in patients undergoing radical cystectomy: A high-volume tertiary cancer center experience. Eur Urol 2009;55(1):177-85.

[11] Lowrance WT, et al. Contemporary open radical cystectomy: Analysis of perioperative outcomes. J Urol 2008; 179(4):1313-8; discussion 1318.

[12] Stimson CJ, et al. Early and late perioperative outcomes following radical cystectomy: 90-day readmissions, morbidity and mortality in a contemporary series. J Urol 2010;184(4): 1296-300.

[13] Ravi P, et al. Is there any evidence of a July effect in patients undergoing major cancer surgery? Can J Surg 2014; 57(2):82-8.

[14] AJCC Cancer Staging Manual. 7 ed. 2010: Springer-Verlag New York. 648

[15] Clavien PA, et al. The Clavien-Dindo classification of surgical complications: Five-year experience. Ann Surg 2009; 250(2):187-96.

[16] Harris PA, et al. Research electronic data capture (REDCap)-a metadata-driven methodology and workflow process for providing translational research informatics support. J Biomed Inform 2009;42(2):377-81.

[17] Harring TR, et al. Liver transplant fellowship and resident training is not a part of the July effect. J Surg Res 2013; 182(1): $1-5$.

[18] Macario A. What does one minute of operating room time cost? J Clin Anesth 2010;22(4):233-6.

[19] Hospital Adjusted Expenses per Inpatient Day. 2015, The Henry J Kaiser Family Foundation.

[20] Sammour T, et al. A programme of Enhanced Recovery After Surgery (ERAS) is a cost-effective intervention in elective colonic surgery. N Z Med J 2010;123(1319):61-70.

[21] Gouvas N, et al. Fast-track vs standard care in colorectal surgery: A meta-analysis update. Int J Colorectal Dis 2009; 24(10):1119-31.

[22] Arumainayagam N, et al. Introduction of an enhanced recovery protocol for radical cystectomy. BJU Int 2008; 101(6):698-701.

[23] Pruthi RS, et al. Fast track program in patients undergoing radical cystectomy: Results in 362 consecutive patients. J Am Coll Surg 2010;210(1):93-9.

[24] Donat SM, et al. Early nasogastric tube removal combined with metoclopramide after radical cystectomy and urinary diversion. J Urol 1999;162(5):1599-602.

[25] Davis JW, et al. Initial experience of teaching robot-assisted radical prostatectomy to surgeons-in-training: Can training be evaluated and standardized? BJU Int 2010;105(8): 1148-54. 